\title{
Desenvolvimento de um aplicativo móvel educacional voltado ao ensino de Estrutura de dados
}

\section{Luiz Ricardo do Carmo de Oliveira, Ian Simon Pinheiro de Souza, Diego da Silva Froner, Aneil Martins de Souza Filho}

\author{
Curso de Ciência da Computação - Centro Universitário do Norte (UNINORTE) \\ luizricardo.co@gmail.com, yansymon@hotmail.com, fronerdiego@gmail.com, \\ aneilmartins@hotmail.com
}

\begin{abstract}
This work presents the creation and the evaluation of an educational app for the study of data structure: The Simplified Analyzer and Algorithm Animation Control (ASCAA). This tool has the purpose to facilitate the teaching of these subjects by using a simple interface. The app contains pseudocodes, descriptions and an interactive animation screen of data structures. $96 \%$ users who participated in ASCAA's evaluation answered positive results, while $100 \%$ considered Very High or High the chances to recommend the app.

Resumo. Este trabalho apresenta a criação e avaliação de um aplicativo educacional para o estudo de estrutura de dados: o Analisador Simplificado e Controle de Animação de Algoritmos (ASCAA). Esta ferramenta tem como propósito tornar o ensino da matéria mais fácil através de uma interface simples. $O$ aplicativo contém pseudocódigos, descrições e uma tela de animação interativa das estruturas de dados. $96 \%$ dos usuários que participaram da avaliação do ASCAA responderam com resultados positivos, enquanto que $100 \%$ consideraram Muito Alta ou Alta a probabilidade de recomendarem o aplicativo.
\end{abstract}

\section{Introdução}

Nos primeiros períodos dos cursos de computação, disciplinas de lógica de programação e estruturas de dados são apresentadas aos estudantes as. Entretanto, para a maioria dos estudantes, este é seu primeiro contato com tais matérias e, levando em consideração que cada estudante possui um tempo próprio de aprendizado, é comum a ocorrência de uma defasagem no entendimento de conceitos básicos desta disciplina. (Pereira, Medeiros, \& Menezes, 2012).

$\mathrm{Na}$ literatura é possível encontrar técnicas de visualização de algoritmos através de animações em ferramentas como o Data Structure Visualization (Galles, 2011) e o JavaTool (Mota, Pereira, \& Favero, 2008). Na primeira é demonstrado o funcionamento de alguns algoritmos de Estrutura de Dados (listas, filas, pilhas , grafos, árvores, ordenação de vetores, entre outros), enquanto que a segunda trata-se de uma ferramenta para o estudo da linguagem de programação Java por meio de uma interface gráfica.

Com o avanço de tecnologias móveis, novas abordagens possibilitam a criação de ferramentas que auxiliem no ensino de uma maneira mais flexível. Visando esta possibilidade, será apresentado neste trabalho um aplicativo para dispositivos móveis com a plataforma Android denominado Analisador Simplificado e Controle de Animação de Algoritmos (ASCAA). O ASCAA apresenta animações de estrutura de dados simples e avançadas, descrições e pseudocódigos. A ferramenta de apoio ao 
ensino ASCAA é composta por estruturas de dados básicas (Fila, Pilha, Tabela Hash), algoritmos de ordenação (Insertion Sort, Bubble Sort, Selection Sort, Quick Sort, Merge

Sort, Shell Sort e Heap Sort), árvores (AVL, Rubro Negra e B) e algoritmos de grafos (Busca em Largura, Busca em profundidade, Dijkstra e Prim). Estes conteúdos foram selecionados para conter no aplicativo por serem recorrentes nas grades curriculares das principais instituições de ensino em Computação do país.

A validação deste trabalho se dará através de questionários voltados para validação de ferramentas educacionais. Participarão desta etapa estudantes com os mais diversos níveis de conhecimento em programação após terem feito uso do aplicativo.

\section{Trabalhos Relacionados}

Foram verificadas através de pesquisas diversas aplicações semelhantes que auxiliam no aprendizado de lógica de programação e estruturas de dados. Estas ferramentas auxiliam no ensino através das mais variadas formas que vão desde compiladores em português, animações de estrutura de dados, criação de algoritmos a partir de objetos gráficos e fluxogramas.

Uma ferramenta que possui uma destas qualidades é o Scratch (MIT, 2007). Esta ferramenta possui uma interface simples projetada para crianças e jovens de 8 aos 16 anos de idade e possui versões online e offline. Suas instruções são definidas através de blocos de comandos pré-prontos nos quais o estudante é livre para definir quais serão suas funcionalidades. (Pereira, Medeiros, \& Menezes, 2012). No entanto, o Scratch é limitado à criação de programas simples, não sendo possível criar programas que exijam um nível de complexidade maior. A Figura 1 apresenta uma visão geral do ambiente de desenvolvimento do Scratch.

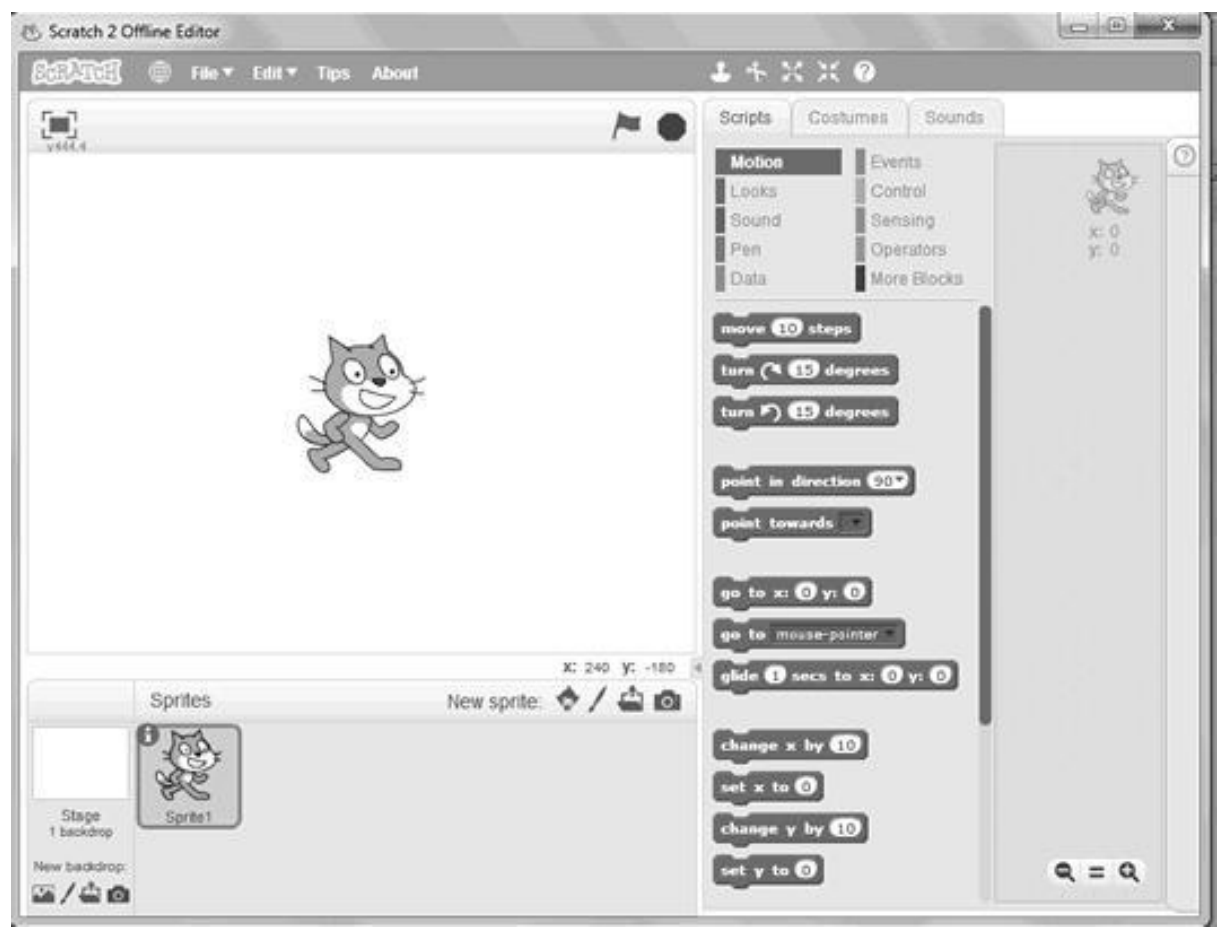

Figura 1. Scratch - Visão geral do Ambiente de Desesenvolvimento.

Outra forma popular de ensinar programação em geral é o uso do Portugol IDE (Manso, Oliveira, \& Marques, 2009). Este ambiente de desenvolvimento integrado é 
V Congresso Brasileiro de Informática na Educação (CBIE 2016)

Anais do XXVII Simpósio Brasileiro de Informática na Educação (SBIE 2016)

utilizado para introduzir os estudantes de programação à construção do raciocínio lógico e sistemático para resolução de problemas computacionais. Sua linguagem de codificação de algoritmo é em português, o que torna bastante intuitivo o entendimento do processo de compilação dos programas. Além da codificação em português, o Portugol IDE possui uma linguagem baseada em fluxogramas, permitindo a construção de programas inteiros através de blocos gráficos. Porém, o Portugol IDE também está limitado a criação de algoritmos simples, não sendo permitido por exemplo, a construção de estruturas de dados como Grafos e Árvores. A Figura 2 mostra a tela de codificação de uma resolução de um problema aritmético de divisão e soma no Portugol IDE.

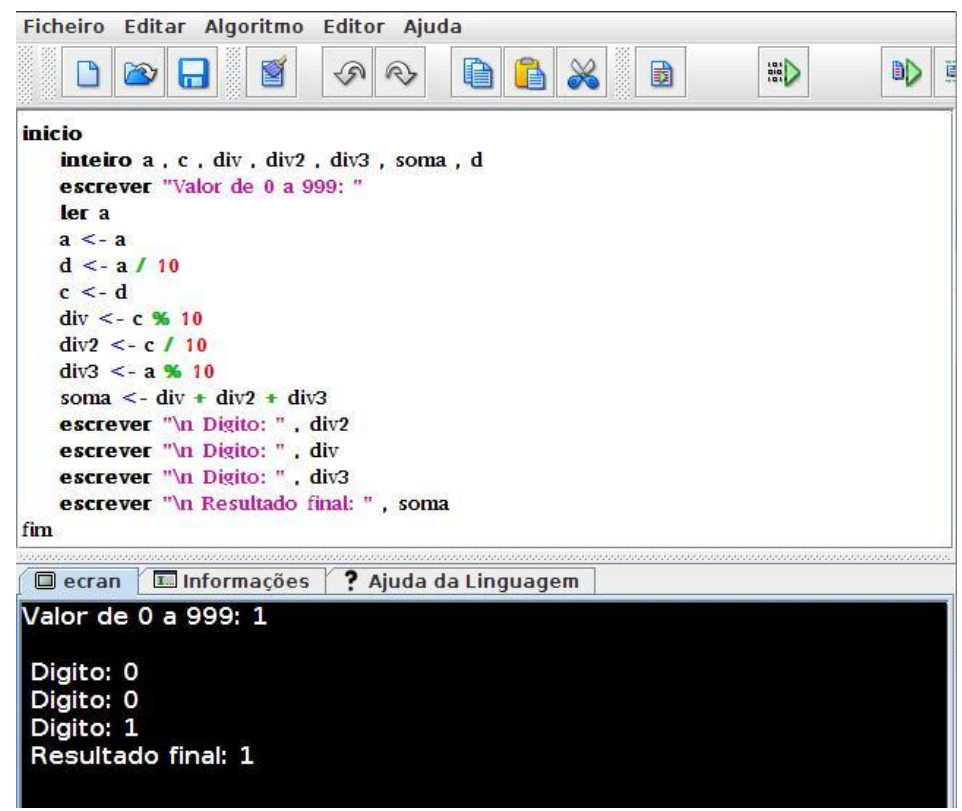

Figura 2. Portugol IDE

Outra ferramenta disponível é o Data Structure Visualization (DSV) (Galles, 2011). Trata-se de um site que possui várias estruturas de dados com representação através de animação. Este Web Visualizador de Estruturas de Dados foi desenvolvido por David Galles da Universidade de São Francisco nos Estados Unidos em 2011. Sua proposta é diminuir a abstração das estruturas de dados através de animações interativas. Apesar da proposta no qual o DSV foi desenvolvido, apenas o estudo das Estruturas de Dados através de animações não é o suficiente para que o estudante possa entender seu funcionamento por completo, deixando assim lacunas no que diz respeito a conceitos das Estrutura de Dados. A Figura 3 demonstra o funcionamento da estrutura de dados FILA no ambiente do DSV. 
V Congresso Brasileiro de Informática na Educação (CBIE 2016)

Anais do XXVII Simpósio Brasileiro de Informática na Educação (SBIE 2016)

\section{Queue (Array Implementaion)}
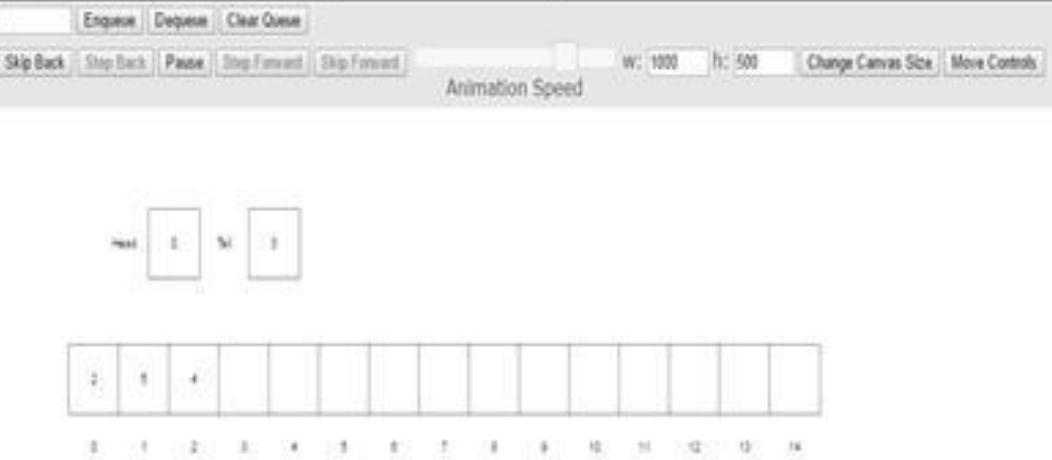

Figura 3. Data Structure Visualization - Animação da estrutura de dados Fila.

Por último, destaca-se também o LEGO MINDSTORM (LEGO, 2016), um Kit desenvolvido pela LEGO Group em parceria com o Media Lab do Massachusetts Institute of Technology (MIT). Seu primeiro Kit Comercial, o LEGO Mindstorm NXT, veio a público em 2006. Desde então, diversas universidades vêm adotando seu uso como parte introdutória nos cursos de graduação de engenharia e computação. No LEGO é possível desenvolver tanto a parte física (montagem do robô) quanto a lógica (definir instruções computacionais através de programas) (Maia, da Silva, Rosa, Junior, \& Neto, 2012). Apesar de os kits do LEGO Mindstorm serem excelentes para a parte introdutória aos estudo de robótica, seu uso fica limitado apenas ao ensino de lógica de programação, não podendo ser usado, por exemplo, ao ensino da matéria de Estrutura de dados ou outra disciplina que envolva conhecimentos mais avançados de programação. As Figuras 4 e 5 mostram a sua versão mais recente, o LEGO Mindstorm EV3.

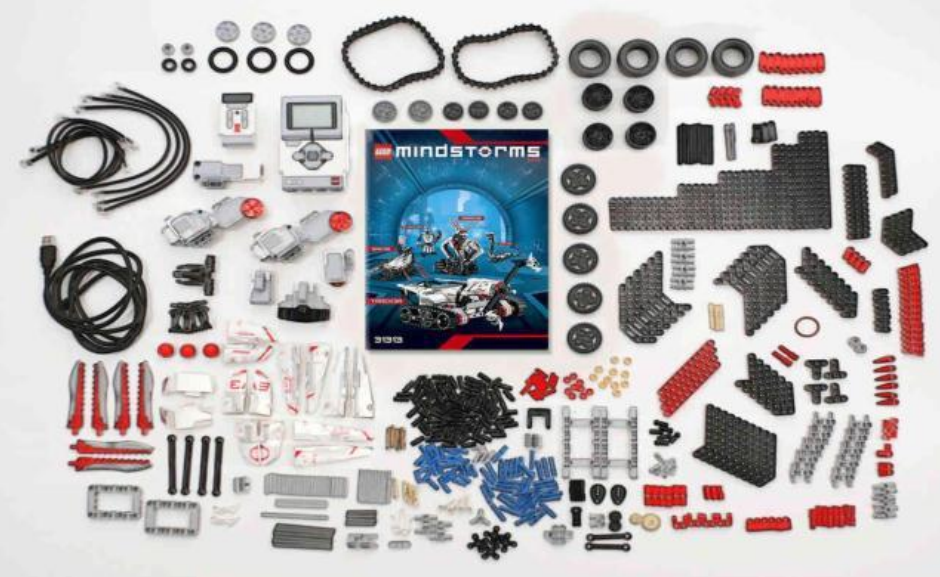

Figura 4. LEGO MINDSTORM EV3 - peças do kit. 


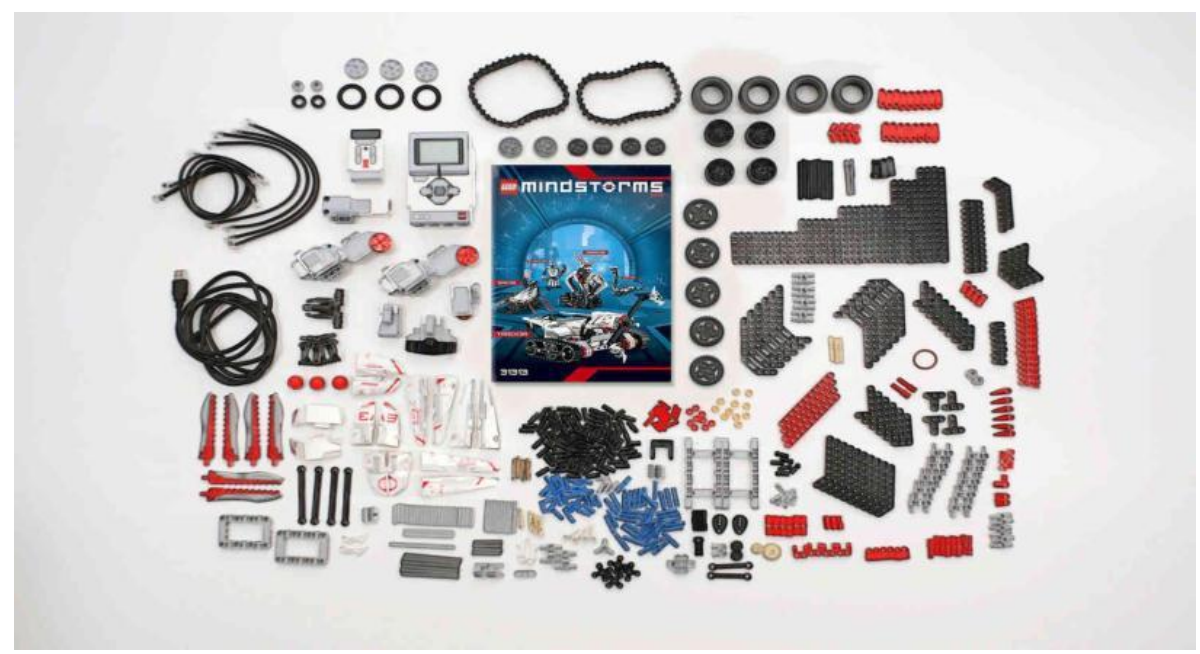

Figura 5. LEGO MINDSTORM EV3 - interface de programação.

\section{Analisador Simplificado e Controle de Animação de Algoritmos}

Uma das maiores barreiras para estudantes de estrutura de dados está em conseguir entender como as estruturas devem funcionar durante a execução dos programas (Galles, 2011).

O ASCAA foi desenvolvido com a finalidade de tornar o ensino da matéria de Estruturas de Dados mais atraente aos estudantes e professores através de uma interface gráfica simples. $\mathrm{O}$ aplicativo apresenta para cada algoritmo uma explicação clara sobre a proposta da estrutura de dados, um pseudocódigo e uma tela com a animação na qual é o estudante pode verificar passo-a-passo como os algoritmos devem funcionar.

A Figura 6 (a) apresenta a tela principal do aplicativo e mostra uma lista das estrutura de dados separadas por tipos. Ao selecionar um item desta lista, o usuário é direcionado para uma tela, como mostrado na Figura 6 (b), na qual é mostrada a descrição da estrutura com seus algoritmos relacionados.

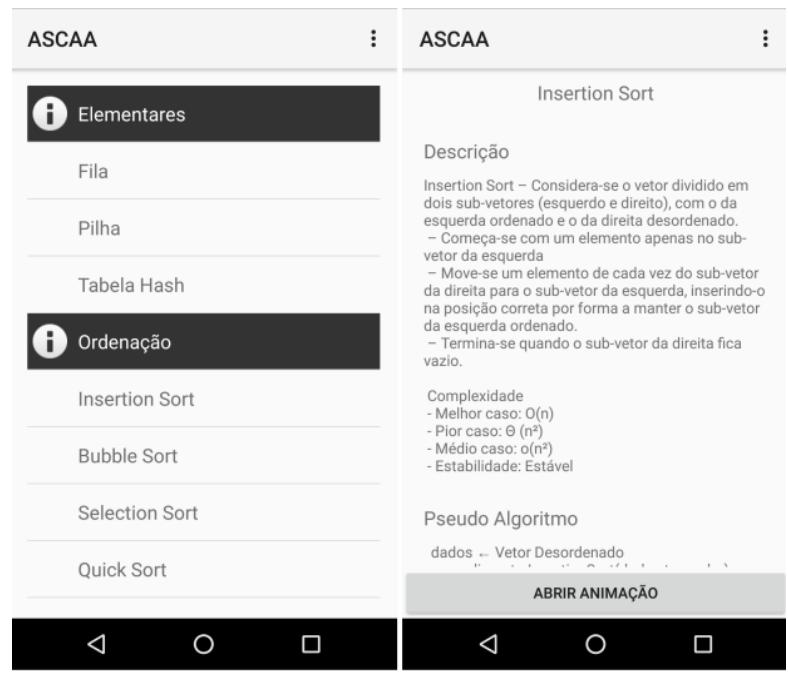

(a)

(b)

Figura 6. (a) - Tela inicial (b) Tela de descrição do algoritmo de ordenação Insertion Sort 
V Congresso Brasileiro de Informática na Educação (CBIE 2016)

Anais do XXVII Simpósio Brasileiro de Informática na Educação (SBIE 2016)

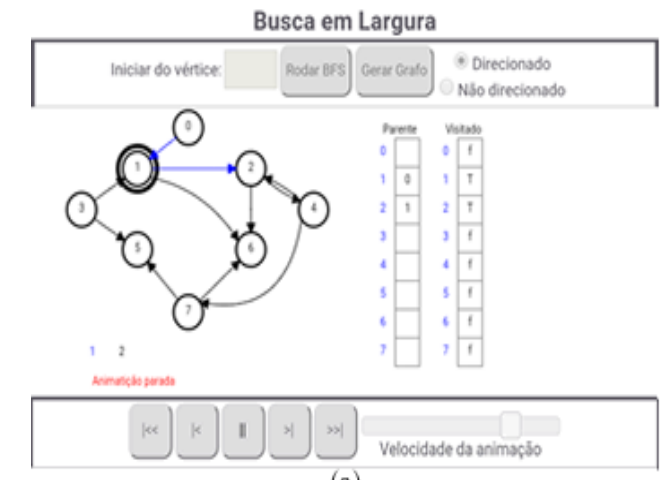

(a)

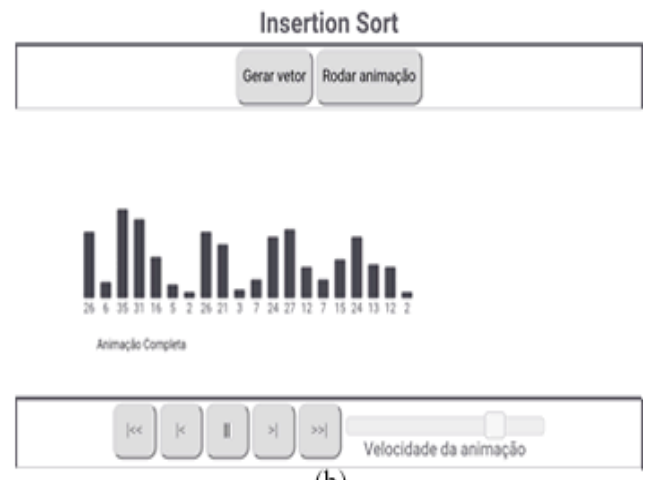

(b)

Figura 7. (a) - Tela de Animação da Estrutura de Dados da Busca em Largura de um Grafo orientado (b) - Tela de Animação do algoritmo de ordenação Insertion Sort.

A Figura 7 mostra a tela de animação do aplicativo ASCAA. Esta parte do aplicativo é interativa, permitindo ao estudante estudar cada passo do processo. Conforme a estrutura de dados selecionado é possível inserir, pesquisar, remover, controlar velocidade da animação, pausar, continuar, voltar ao estado inicial, retroceder passos, avançar passos e pular para o estado final.

\section{Avaliações}

Foram realizados testes com 30 estudantes nos mais variáveis níveis de conhecimento em programação dos cursos de Ciência da Computação, Engenharia da Computação e Tecnologia em Análise de Sistemas do Centro Universitário do Norte (UNINORTE).

As avaliações aconteceram em duas etapas com os mesmos usuários sem um limite de tempo para a realização. Na primeira situação, os estudantes foram submetidos a realizar passos pré-definidos, enquanto que na segunda situação estes mesmos usuários exploraram o aplicativo livremente. A Figura 8 mostra os resultados obtidos no questionário respondido pelos usuários após o término das duas etapas.

Os passos da primeira situação foram os seguintes:

- Abrir o aplicativo;

- Ir a estrutura de dados FILA;

- Inserir 4 valores;

- Remover 2 valores;

- Esvaziar a FILA;

- Voltar à tela inicial

O resultado da pesquisa do aplicativo ASCAA mostrou um índice de $96 \%$ se somados as porcentagens das notas acima de médio (Muito alto, alto, bom, ótimo e muito conhecimento) e 4\% para notas consideradas baixas (Baixo, Muito Baixo, Regular, Ruim, Péssimo e Pouco, Médio ou Nenhum Conhecimento), resultando em uma ampla aceitação do uso do aplicativo ASCAA como ferramenta de apoio ao ensino de Estrutura de dados e Lógica de programação. 
V Congresso Brasileiro de Informática na Educação (CBIE 2016)

Anais do XXVII Simpósio Brasileiro de Informática na Educação (SBIE 2016)

\begin{tabular}{|c|c|c|c|c|c|c|c|c|c|c|c|c|c|}
\hline \multicolumn{2}{|c|}{$\begin{array}{l}\text { - Como você } \\
\text { considera o seu } \\
\text { conhecimento na } \\
\text { matéria que o } \\
\text { aplicativo aborda }\end{array}$} & \multicolumn{2}{|c|}{$\begin{array}{l}2 \text { - } 0 \text { quanto você considera que o } \\
\text { aplicativo ASCAA contribui para o } \\
\text { aumento do conhecimento em } \\
\text { Lógica de Programação e Estrutura } \\
\text { de Dados? }\end{array}$} & \multicolumn{2}{|c|}{$\begin{array}{l}3 \text { - Em sua opinião, } \\
\text { em quanto o uso do } \\
\text { aplicativo ASCAA, } \\
\text { pode contribuir com a } \\
\text { motivação para a } \\
\text { aprendizagem em } \\
\text { Logica de } \\
\text { Programação e } \\
\text { Estrutura de Dados? }\end{array}$} & \multicolumn{2}{|c|}{$\begin{array}{l}4 \text { - Qual a } \\
\text { probabilidade de você } \\
\text { recomendar o } \\
\text { aplicativo ASCAA } \\
\text { para outra pessoa? }\end{array}$} & \multicolumn{2}{|c|}{$\begin{array}{l}5 \text { - Indique o nivel que } \\
\text { representa a facilidade } \\
\text { de uso do Aplicativo } \\
\text { ASCAA em sua } \\
\text { opinião. }\end{array}$} & \multicolumn{2}{|c|}{\begin{tabular}{|l|}
$6-0$ Aplicativo \\
ASCAA apresentou \\
alguma falha durante \\
o uso?
\end{tabular}} & \multicolumn{2}{|c|}{$\begin{array}{l}7 \text { - Qual é a sua } \\
\text { opinião geral sobre o } \\
\text { Aplicativo ASCAA? }\end{array}$} \\
\hline Muito alto & $3.0 \%$ & Muito conhecimento & $73 \%$ & Muito alto & $53 \%$ & Muito alto & $56 \%$ & Muito alto & $56 \%$ & Sim & \begin{tabular}{l|l}
$0 \%$ & $(1$ \\
\end{tabular} & Ótimo & $72 \%$ \\
\hline Alto & $23.0 \%$ & Médio conhecimento & $20 \%$ & Alto & $43 \%$ & Alto & $44 \%$ & Alto & $30 \%$ & Não & \begin{tabular}{l|l}
$100 \%$ & $\mathrm{~B}$ \\
\end{tabular} & Bom & $21 \%$ \\
\hline Médio & $60.0 \%$ & Pouco conhecimento & $0 \%$ & Médio & $0 \%$ & Médio & $0 \%$ & Médio & $10 \%$ & & & Regular & $7 \%$ \\
\hline 3aixo & $12.0 \%$ & Nenhum conhecimento & $7 \%$ & Baixo & $4 \%$ & Baixo & $0 \%$ & Baixo & $4 \%$ & & & Ruim & $0 \%$ \\
\hline Muito Baixo & $2.0 \%$ & & & Muito Baix & $0 \%$ & Muito Baixo & $0 \%$ & Muito Baixo & $0 \%$ & & & Péssimo & $0 \%$ \\
\hline
\end{tabular}

Figura 8. Resultados dos questionários respondidos pelos estudantes após o uso do aplicativo

Notou-se também que no item 4, referente a recomendação do aplicativo a outras pessoas, $56 \%$ responderam a opção Muito Alto, $44 \%$ a opção Alto e nenhum participante respondeu abaixo disto.

De modo geral, $72 \%$ avaliaram o aplicativo ASCAA como Ótimo, $21 \%$ como bom e 7\% como regular, obtendo $0 \%$ para resultados Ruim e Péssimo. Conclui-se com

estes resultados que o uso do aplicativo ASCAA como ferramenta de apoio ao ensino esta dentro dos padrões de aceitação dos alunos, podendo ser utilizados por professores como auxílio ao ensino da matéria de estrutura de dados.

\section{Considerações finais e Trabalhos Futuros}

Desenvolver o aplicativo móvel ASCAA (Analisador Simplificado e Controle de Animação de Algoritmos) foi um grande desafio e exigiu uma pesquisa elaborada. Após a avaliação dos motivos para os altos índices de reprovação e desistência dos cursos de computação, constatou-se que as dificuldades enfrentadas no aprendizado das disciplinas de estruturas de dados são um dos maiores motivos para ambas as situações. A utilização do aplicativo ASCAA como ferramenta de apoio ao ensino mostrou ser bastante eficiente de acordo com os resultados da pesquisa de satisfação realizada e espera-se que seu uso seja cada vez mais frequente entre os estudantes de universidades como ferramenta de apoio ao ensino de estrutura de dados.

Ao testar o aplicativo ASCAA, alguns ítens foram levantados como sugestão de melhorias e incremento a ser inclusas nas próximas versões. Um destes itens é a inclusão de mais algoritmos de estruturas de dados para estudo de teoria dos grafos. Foi constatado que esta disciplina também necessita de um conhecimento avançado de estrutura de dados e o aplicativo possui potencial para esta abordagem. Outro ítem levantado por muitos estudantes está em ajustes no layout da tela de animação. Conforme relatado por alguns usuários, a resolução apresentou problemas nas telas de alguns dispositivos. Por último, está uma sugestão de melhoria em relação a tela de animação nas estruturas de dados que requerem ações de inserção, remoção e pesquisa de valores. Alguns participantes propuseram a inclusão de uma opção para a geração de valores aleatórios como entrada para as estruturas de dados além da portabilidade do aplicativo ASCAA a outras plataformas móveis, tais como IOS e Windows Phone. 
V Congresso Brasileiro de Informática na Educação (CBIE 2016)

Anais do XXVII Simpósio Brasileiro de Informática na Educação (SBIE 2016)

Como trabalhos futuros propõe-se a adoção oficial do aplicativo para disciplinas de programação e estruturas de dados durante todo um semestre letivo. O objetivo deste estudo é comparar os índices de aprendizagem em uma turma que fez uso do ASCAA com os de outra turma onde apenas métodos tradicionais de ensino foram abordados.

\section{Referências}

Galles, D. (2011). About us Data Structure Visualization. - Disponível em $<$ https://www.cs.usfca.edu/ galles/visualization/about.html>. Acesso em 20 de março de 2016.

Maia, L. D., da Silva, V. J., Rosa, R. E., Junior, V. F., \& Neto, J. P. (2012). A Robótica como Ambiente de Programaçao Utilizando o Kit Lego Mindstorms. XL Congresso Brasileiro de Educação em Engenharia (p. 2). Belém: COBENGE.

Manso, A., Oliveira, L., \& Marques, C. G. (2009). Portugol IDE - Uma ferramenta para o ensino de programação.

MIT. (2007). About us: Scratch. - Disponível em <https://scratch.mit.edu/about/>. Acesso em 15 de março de 2016.

Mota, M. P., Pereira, L. W., \& Favero, E. L. (2008, Junho 12). JavaTools Uma ferramenta para ensino de programação. Workshop sobre Educação em Computação, p. 2.

Pereira, P. d., Medeiros, M., \& Menezes, J. W. (2012, Setembro 3). ANÁLISE DO Scratch como ferramenta de auxílio ao ensino de programação de computadores.

XL Congresso brasileiro de educação em Engenharia, p. 2. 